Monatsschr Kinderheilkd

2020 168 (Suppl 3):S188-S196

https://doi.org/10.1007/s00112-020-00949-1

Online publiziert: 28 . Juli 2020

(c) Springer Medizin Verlag GmbH, ein Teil von Springer Nature 2020

\section{Einführung}

Am 19.04.2018 erschien in der psychiatrischen Fachzeitschrift Molecular Autism der Beitrag „Hans Asperger, National Socialism, and ,race hygiene' in $\mathrm{Na}$ zi-era Vienna“ des Wiener Historikers Czech [11]. Die Hauptaussage wurde in einem Satz zusammengefasst: „The narrative of Asperger as a principled opponent of National Socialism and a courageous defender of his patients against Nazi ,euthanasia' and other race hygiene measures does not hold up in the face of the historical evidence." Zehn Tage später veröffentlichte die US-amerikanische Historikerin Sheffer ihr Buch [33], in dem sie z.T. dasselbe Material wie Czech verwendet und ein ähnliches, insgesamt jedoch beträchtlich vergröbertes Bild zeichnet. Die Publikationen wurden im vorangehenden Editorial in Molecular Autism dahingehend interpretiert, dass „like Czech, Sheffer compellingly makes the case that Asperger willingly became a cog in the Nazi killing machine" [7].

Die internationale Presse reagierte für den wissenschaftlichen Diskurs ungewöhnlich schnell auf die beiden Publikationen, teils mit Berichten, die noch direkt am Erscheinungstag veröffentlicht wurden und das Zerrbild eines „karrieristischen, den Tod von Kindern akzeptierenden Monsters" entwarfen ([9, 16, 28, 29, 38]; - Tab. 1). Obwohl sich die diversen Boulevardzeitungen auf die Arbeiten von Czech und Sheffer beriefen, erfolgte auf diese teils unseriöse und tendenziöse Berichterstattung von den beiden HistorikerInnen gemäß Wissen der Autoren des vorliegenden Beitrags

Franz Waldhauser ${ }^{1}$ Johann Deutsch · Christian Popow

'Wien, Österreich

\title{
Hans Asperger - Zwischenbericht einer Aufarbeitung durch seine SchülerInnen und KollegInnen
}

keine Richtigstellung oder zumindest adäquate Distanzierung.

Genau genommen gab es allerdings bereits frühere Berichte über Aspergers angebliche Verflechtung mit dem Nationalsozialismus (NS). Czech etwa wies schon vor mehreren Jahren im Rahmen eines Interviews auf Aspergers bis dahin recht unbekannte, potenzielle Kollaboration mit dem NS-Regime hin [18]. Dem Interview voran ging bereits eine entsprechende, jedoch deutschsprachige Publikation [10].

Der US-amerikanische Autor Donvan versuchte sich im Rahmen des internationalen Diskurses 2018 an einer Interpretation, warum Aspergers Arbeit während der NS-Zeit nicht bereits früher beforscht wurde, und vermutete geografische und sprachliche Gründe. Immerhin hatte Asperger nie auf Englisch publiziert und praktisch seine gesamte Karriere in Österreich verbracht. Er zeigte aber auch Details über Motivation, Vorbereitung, Zielsetzung und z.T. Ablauf der von der internationalen Presse vorgenommenen Aktion gegen Asperger ([13]; • Tab. 1).

Nach einer anfänglichen Schockphase begannen die Autoren des vorliegenden Beitrags, einige ehemalige SchülerInnen, später oft auch KollegInnen von Asperger, die inzwischen meist 70 und teils mehr als 90 Jahre alt sind, zu diskutieren, ob dies der von ihnen gekannte Hans Asperger sein könnte? Konnte dieser literarisch gebildete, zutiefst humanistisch und katholisch geprägte Mensch in früheren Jahren ein karrieristisches, über andere hinwegtrampelndes Zerrbild seiner selbst gewesen sein, das sich aktiv an Hitlers Rassenhygiene beteiligt und kranke Kinder wissentlich in den Tod geschickt hatte? In der Erinnerung seiner ehemaligen SchülerInnen und KollegInnen war er doch ein nie jemanden beleidigender oder drängender Mensch, der eher durch Fragen und Meinungen sanft, vielleicht manchmal zu sanft, $\mathrm{zu}$ stimulieren versucht hatte. Wann sollte dieser Bruch erfolgt, diese Wandlung geschehen sein?

War er vielleicht für seine damals junge Familie Kompromisse eingegangen und hatte dabei unter Druck Zwangssterilisation und Euthanasie in Kauf genommen? Hatte er seine SchülerInnen und KollegInnen getäuscht, und hatten diese sich über Jahrzehnte täuschen lassen?

\section{》) Gerade für die älteren KollegInnen war Asperger weder ein Nazi noch ein Nazikollaborateur}

Gerade die Älteren, die lange mit ihm zusammengearbeitet haben, sagen ein klares „Nein“. Für sie war Asperger weder ein Nazi noch ein Nazikollaborateur. Unvorstellbar!

Die Autoren begannen also den in den beiden Publikationen von Czech und Sheffer angeführten Argumenten nachzugehen. Wie solide sind diese?

\section{Bund Neuland}

Asperger war sein Leben lang ein überzeugter Neuländer (Artikel von Maleczek et al. im vorliegenden Sonderheft). In einem Radiointerview zu Weihnachten 1974, knapp 6 Jahre vor seinem Tod, zi- 
Tab. 1 Auszug aus der weltweiten Berichterstattung vom Tag bzw. Folgetag des Erscheinens des Beitrags über Asperger in Molecular Autism

\begin{tabular}{|c|c|c|c|}
\hline Datum & Medium & Titel & Referenz \\
\hline 19.04.2018 & Molecular Autism & Czech H: „Hans Asperger, National Socialism, and ,race hygiene’ in Nazi-era Vienna” & {$[42]$} \\
\hline 19.04.2018 & Molecular Autism & Editorial: Did Hans Asperger actively assist the Nazi euthanasia program? & {$[43]$} \\
\hline 18.04.2018 & The Guardian & Hans Asperger aided and supported Nazi programme, study says & [44] \\
\hline 19.04.2018 & New York Times & Hans Asperger aided Nazi child euthanasia, study says & {$[45]$} \\
\hline 19.04.2018 & Washington Post & $\begin{array}{l}\text { Hans Asperger, hailed for autism research, may have sent child patients to be killed by } \\
\text { Nazis }\end{array}$ & [46] \\
\hline 19.04.2018 & Telegraph & Pioneer doctor Asperger was Nazi collaborator who „sent children to their deaths" & {$[47]$} \\
\hline 19.04.2018 & Stuff (New Zealand) & Child doctor Hans Asperger „willingly became cog in the Nazi killing machine” & [48] \\
\hline 20.04.2018 & SBS News (Australia) & $\begin{array}{l}\text { Austrian paediatrician Hans Asperger reportedly "actively cooperated " with the Nazi } \\
\text { euthanasia programme }\end{array}$ & {$[49]$} \\
\hline 19.04.2018 & $\begin{array}{l}\text { South China Morning- } \\
\text { Post }\end{array}$ & $\begin{array}{l}\text { Famed paediatrician Hans Asperger "actively cooperated“ with Nazis during second } \\
\text { world war, according to new research }\end{array}$ & {$[50]$} \\
\hline 19.04.2018 & Folha de S.Paulo (Brasil) & Asperger colaborou com programa de eutanásia nazista, diz estudo & {$[51]$} \\
\hline 19.04.2018 & Die Zeit & Hans Asperger: Ein guter Nazi? & [52] \\
\hline 19.04.2018 & Neue Zürcher Zeitung & Hans Asperger soll Kinder an die Nazis ausgeliefert haben & {$[53]$} \\
\hline 19.04.2018 & Der Standard & Autismusforscher Hans Asperger schickte Kinder in den Tod & {$[54]$} \\
\hline 20.4.2018 & Die Presse & Asperger half der NS-Euthanasie & {$[55]$} \\
\hline 01.05 .2018 & Buch & Sheffer E: „Asperger's children: the origins of autism in Nazi Vienna” & {$[56]$} \\
\hline
\end{tabular}

tierte er den Kern der „Meißner-Formel“, gewissermaßen das "Glaubensbekenntnis“ der Neuländer,: Sie wollen „nach eigener Bestimmung, vor eigener Verantwortung, in innerer Wahrhaftigkeit ihr Leben gestalten“. In den Folgeminuten versuchte er, die Bestrebungen des Bund Neuland darzulegen und den Unterschied zu anderen Gruppierungen der politischen Jugendbewegung zu skizzieren: „Die politische Jugendbewegung aller Färbungen, aber besonders die HJ [Hitlerjugend; Anm. d. Autoren], hat eben gerade das Wesentliche, das aus innerer Freiheit, aus echter Verantwortung, das Richtige zu tun, das Leben der Jugend zu führen, ins Gegenteil verkehrt und war eine schreckliche Schule der Unfreiheit" [3].

I) Asperger gehörte zu den "Fahrenden Scholaren", deren Gründer Hesse von der Gestapo verbannt wurde

Die Autoren kontaktierten den Historiker Seewann $\left({ }^{*} 1944\right)$, den Verfasser des Standardwerks über den Bund Neuland [30, 31], und befragten ihn zur Darstellung des Bund Neuland in Czechs Publikation. Seewanns zusammengefasste
Antwort: So nicht, und übrigens habe er selbst Asperger damals bei der Abfassung seiner Publikation als integre Person kennengelernt und werde ihm für die Erfahrung dieses Gesprächs ein Leben lang dankbar sein (persönliche Mitteilung vom 06.08.2018).

Die genauere Auseinandersetzung mit dem Bund Neuland begann: Waren die Neuländer denn affin für den damals erstarkenden NS? Einige waren Mitglieder der ab Juni 1933 illegalen österreichischen NSDAP, wie etwa ihr Bundesführer Anton Böhm (1904-1998; [8, S. 188]), was dieser zeitlebens vehement bestritt [21, S. 182-184]. Er und manche andere sind jedenfalls sicher 1938 Nazis geworden, hier v. a. die Jüngeren, die erst in den 1930-Jahren zu dieser Bewegung gestoßen sind; andere, hier nun v.a. die in den 1920-Jahren Beigetretenen, wiederum nicht. Letztere sind z.T. von den Nationalsozialisten bis hin zur Verschleppung ins Konzentrationslager verfolgt worden, wie die Politiker Felix Hurdes (1901-1974) und Lois Weinberger (1902-1961) oder die Priester Erwin Hesse (1907-1992) und Otto Mauer (1907-1973; [21, S. 162]). Es hing wohl also sehr davon ab, wann man zum Bund Neuland gestoßen war und zu welcher der sehr unterschiedlichen und eigenständigen Untergruppen man gehörte [21, 30, 31].

Asperger war bei den „Fahrenden Scholaren“, die von Czech als „part of the Bund's decidedly völkisch and rightwing faction" bezeichnet werden; Evidenz bringt er für diese Aussage keine. Tatsächlich gründete der (schon erwähnte) 16-jährige Erwin Hesse im April 1923 die „Fahrenden Scholaren“ [6]. Er wurde nach dem sog. Anschluss von der Gestapo aus Wien verbannt, war Mitglied der „Kirche im Widerstand“, letztlich Pfarrer von St. Rochus und später Prälat [24, 39]. Was sie damals dachten und taten, schrieb Hesse in mehreren Aufsätzen in den frühen 1920er-Jahren nieder. In der Brünner Verbannung editierte er diese für seinen Bruder [20]. In einem zentralen Aufsatz wird ihr Leitbild festgehalten (- Abb. 1).

Asperger schrieb damals über Hesse: „Er hat uns gerufen, er hat den Gau [gemeint sind die, Fahrenden Scholaren'; Anm. d. Autoren] gegründet, er konnte immer am besten sagen, was wir wussten, was wir wollten und was wir sollten" [6]. Und er leitete mit Hesse gemeinsam die „Fahrenden Scholaren“. Neben ihren Wanderungen und Fahrten in die Natur arbeiteten sie intensiv mit Michael Pfliegler (1891-1972) an der Reform des „Christlich-Deutschen Studen- 
Monatsschr Kinderheilkd 2020 • 168 (Suppl 3):S188-S196 https://doi.org/10.1007/s00112-020-00949-1

(c) Springer Medizin Verlag GmbH, ein Teil von Springer Nature 2020

\section{F. Waldhauser ·. Deutsch · C. Popow}

\section{Hans Asperger - Zwischenbericht einer Aufarbeitung durch seine Schülerlnnen und Kolleglnnen}

\section{Zusammenfassung}

In zwei rezenten Publikationen wurde Hans Asperger, dem Erstbeschreiber des später nach ihm benannten Syndroms, unterstellt, in der Zeit zwischen 1938 und 1945 mit dem nationalsozialistischen (NS-)Regime bei Zwangssterilisation und Euthanasie zusammengearbeitet zu haben. Die internationale Presse hat, sich teils berufend auf diese Berichte, aus Asperger ein „karrieristisches, den Tod von Kindern akzeptierendes Monster" kreiert, ohne dass die verantwortlichen Autorlnnen nach Wissen der Autoren des vorliegenden Beitrags in adäquater Form protestiert hätten.

Da sich das gezeichnete Bild mit den Erfahrungen von zahlreichen seiner noch lebenden Schülerlnnen und Kolleglnnen nicht deckte, wurde von diesen im ersten Schritt folgenden der publizierten Äußerungen nachgegangen:

1. Angaben zu den „Fahrenden Scholaren“, einer Gruppe in der katholischen Jugendorganisation „Bund Neuland", die Asperger mit dem späteren katholischen Priester Erwin Hesse als Mittelschüler leitete und zu deren Ideen er lebenslang stand.

2. Darstellung der St.-Lukas-Gilde, einer katholischen Ärztegruppe, die in den 1930erJahren gegen die nationalsozialistisch propagierte Idee von Zwangssterilisation und Rassenhygiene aufgetreten ist, und in deren Vorstand Asperger war.

3. Darstellungen, nach denen Asperger die Unwahrheit über seine Verfolgung durch die Gestapo gesagt haben soll.
Bereits in diesen Punkten liegen aus der Sicht der Autoren gravierende Unterschiede zwischen bekannten Fakten und der Darstellung, meist durch Weglassungen oder Interpretationen, vor. Entsprechend haben sich 21 von Aspergers Schülerinnen und KollegInnen um eine Gegendarstellung im primären Publikationsorgan bemüht, die jedoch von den HerausgeberInnen abgelehnt wurde. Zudem wurde gemeinsam mit Historikern eine Arbeitsgruppe gebildet, die den kürzlich publizierten Darstellungen nachgeht. Erste Ergebnisse werden im Folgenden vorgelegt; weitere sind in Ausarbeitung.

\section{Schlüsselwörter}

Nationalsozialismus · Zwangssterilisation . Euthanasie · Katholizismus · Arztrolle

\section{Hans Asperger-Interim report of a processing by his students and colleagues}

\section{Abstract}

In two recent publications on Hans Asperger, the first describer of a syndrome that was later named after him, it has been insinuated that he cooperated with the National Socialist (NS) regime's forced sterilization and euthanasia programs during the period 1938-1945. The international press, partly relying on these reports, has portrayed Asperger as a "careerist monster accepting the death of children", to the knowledge of the authors without adequate protest by the responsible authors. As the fabricated image does not match the experiences of many of his still living students and colleagues, some of the published statements were investigated as a first step:
1) Information on the "Fahrende Scholaren" (Traveling Scholars), a subgroup of the catholic youth organization "Bund Neuland," which Asperger led as a high school student together with future Catholic priest Erwin Hesse, and whose tenants he followed his whole life. 2) The portrayal of the "St.-Lukas-Gilde" (Guild of Saint Luke), a Catholic group of physicians who adamantly opposed the ideas of forced sterilization and racial hygiene propagated by the National Socialists in the early 1930 s, and in which Asperger was a member of the board. 3) The portrayal that Asperger allegedly lied about his persecution by the Gestapo. Due to the, in our opinion, serious differences between the known facts and the portrayal of these few points, mostly due to omission or interpretation, 21 students and former colleagues of Asperger presented a counter statement to the primary publication organ, which was, however, refused by the editors. Together with historians, a working group has been formed to investigate the recently published portrayal. Some preliminary findings are presented here, while other results are still being processed.

Keywords

National socialism · Sterilization, involuntary . Euthanasia · Catholicism · Physician's role tenbundes“ und an der Gründung von „Jungösterreich“, der Vorgängerorganisation vom Bund Neuland [30, S. 207]. Asperger meinte 1934 zu dieser Zeit: „Wir fanden Österreich, wir haben mit seinen Bergen gerauft, wir kennen seine stillsten Täler, im erwachenden Frühling, aber auch an langen Regentagen, wo alles groß und einsam ist. Wir kennen Österreichs Menschen und ihre Lieder, wir wissen um den schweigenden Adel Stifterischer ${ }^{1}$ Gestalten“ [6].

Diese Entwicklungen bei den „Fahrenden Scholaren“ geschahen wenige Jahre, nachdem die politischen Parteien Deutschösterreichs für einen Anschluss „Restösterreichs“ an die Weimarer Republik votiert hatten, in der Annahme, dass „Restösterreich“ allein nicht über-

\footnotetext{
1 Adalbert Stifter (1805-1868), österreichischer Dichter des Biedermeier.
}

lebensfähig wäre [19, S. 334-346, 23, S. 43,36, S. $12-13$ ]

\section{St.-Lukas-Gilde}

Eine andere Frage, der nachgegangen wird: Konnte sich Asperger tatsächlich durch seine Mitgliedschaft bei der 1932 in Wien gegründeten St.-Lukas-Gilde das Vertrauen der NSDAP erwerben, wie in den Raum gestellt wird [11, S. 6].

Diese Vereinigung katholischer ÄrztInnen vertrat zahlreiche katholische Zie- 


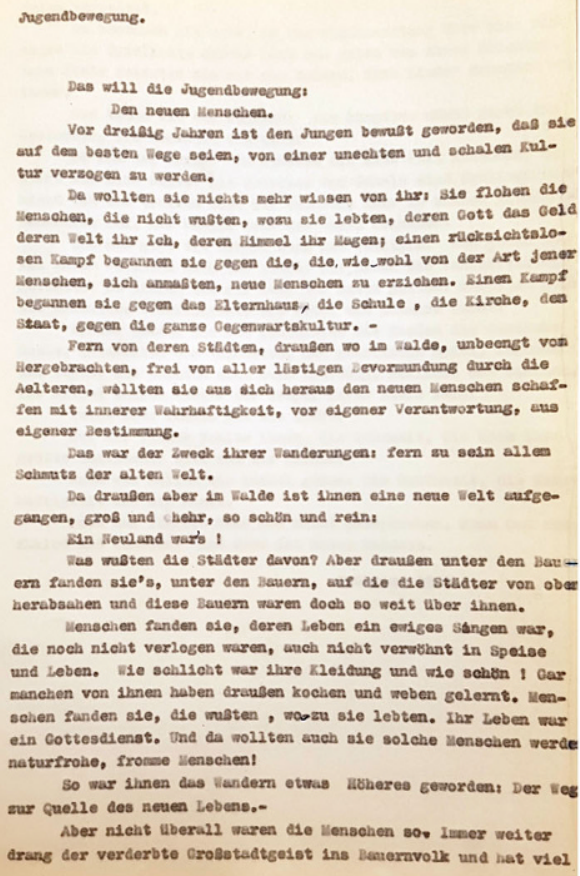

resenaberasung.

Das w112 a1e Jugendberagung

Vor dresilg Jahren lat den Jungen berrubt goworcen, das ale ous dea besten iege selen, von elner unechten und schalen kultur verzogen au werien.

De wollten sie niehts mehr wiseen von lhyi s1e Rlohen 010 ohen, Ale nioht mubten, mosu 10 lebten, deren cott das celd

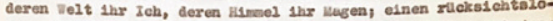

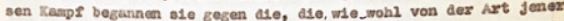
Uensohen, sloh ansusten, neue Jensohen su ersiohen. Binen kamps begannen sie gegen dag Z1ternhaus, O1e schule, A1e K1rohe, dea steat, Eogen ale ganse Cegenrartakultur.

Fern von ceren stelaten, drauken wo 1n waleo, unbeongt vo lexgebrachten, fre1 von eller listigen zevornundung furch ale Aelteren, wollten a1e aus ilch heraus den neuen Hensohen schasfen m1t 1nnerer Wairhaft1gike1t, vor e1gener Verantimortung, ate

eigener zestineung.

Das war der zreck threr vanderungent fern au sein allem

tis cer alten welt.

Da druaben aber im walde 1st linen eine neue velt uargeangen, ExOB und chehr, so schth und rein

Sin Ieviand warts :

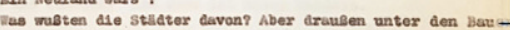
exn fanden s1e's, unter den Bauern, aus die die studter von obo herabsahen and diese Dauesn waren dooh so wo1t uber lhnen. uenseien fanden o1e, doren heben ein erriges singen war, a1e noch nielit verlogen waren, wuch nloht verwohnt in Speise

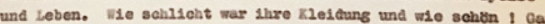

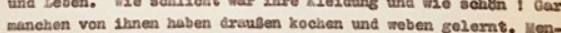

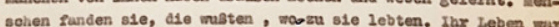

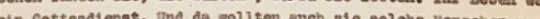
ein cottesdienst. Und dis woy.

So war Innen das tandern

zur cuelze des neuen Lebens. Aber nicht uberall waren d10 llensehen so, Imeer weiter drang cor vercerbte Grosetadtgeist ins Bearernvolk und hat v1e

Cutes verpestot.

Da besannen a10 sich, da kam Verantwortung uber sie: sio zogen als Splellebte durchs Lund und gaben von ihrem Relohtum. Thre Feste Pelerten gio mit Ihnen.

Und taten wie der Heiland: s1e klapsten nicht gegen das Schlechte, sie brachten das cutel

Da kam Cer Kriog: Da sturete die alte Welt zuaamicn, es starb der alte Gelst! Die Wensohen von damals sind freilich noc nioht tot und treiben noch $1 \mathrm{hr}$ Jnwesen, Aber in vielen Elimat Seansuoht auf, ein iunger nach den neven kensehen. Und nun gilt die Arbe1t auch Cen Stliatern. Nun geben s1e von Ihrem aleichtum auch der stadt! sie haben die wahrhartigke1t gefunden! Da ließen sie nur nehr das Echte gelten! Klimgsten geg Gie unleutere Geselligkeitp oie das Echte gelten! Klimperten Sphter fanden s1e die Schunhelt; sle fanden die doutsche Seele, entaeckten das Valkslied, das gelat21che Splel, Legende und sagen. S1e schuren eine noue Klelaung, sehlleht und einfach. Ihr Kurper sollte wieder der Zempel linrer sehlieht Jetzt aber fanden ale die Liebe.
Jelle Wur der claube fohlte lhnen, die Wahrhe1t, die doch inre Crobite Selansucht! Gott und die Kirohe Auch w1r wollen ale suchen gehen, D1e Schlnhest, ale wahr haftigke1t und die Liebe.

Doch das Ilerste kann man nicht susspreohen, kann man nur ruhlea und schsuen: tone dszu lot unser wandern.

\section{Fruhling 1923.}

le mit Bezug zur Medizin [41] und reagierte u. a. auf den wissenschaftlichen Eugenikdiskurs. Sie vertrat die katholische Position im Sinne einer strikten Ablehnung von Sterilisation und Zwangssterilisation und kam damit in krassen Widerspruch zu den NS-Ideen $[17,26]$. Höhepunkt dieses Engagements war der im Frühjahr 1936 in Wien organisierte „Internationale Kongress katholischer Ärzte", bei dem, auf besonderen Wunsch von Pius XI. als erstes Hauptthema „Eugenik und Sterilisierung " diskutiert wurden $[17,40]$.

Entsprechend waren die ProtagonistInnen nach dem sog. Anschluss der Verfolgung durch das NS-Regime ausgesetzt. Bereits am 23.03.1938 verhaftete die Gestapo die am histologischen Institut der Universität Wien tätige Carla ZawischOssenitz (1888-1961), die Mitgründerin und Sekretärin der Gilde sowie spätere Chefredakteurin der Gilden-Zeitschrift St. Lukas. Mitteilungen der österr. St.-Lukas-Gilde war. Wegen ihrer Tätigkeit bei der Gilde wurde ihr die Lehrbefugnis entzogen und ihre Stelle an der Universität nicht verlängert. Nur durch Flucht entging sie weiteren $\mathrm{zu}$ befürchtenden Verfolgungen; in Abwesenheit wurden ihr schließlich die Staatsbürgerschaft und das Doktorat aberkannt $[22,27] .^{2}$

\section{) Mehr als 10\% Prozent der Gildenmitglieder wurden von den Nazis verfolgt}

Ähnlich, aber nicht so drastisch, erging es dem Pädiater Herbert Orel (1898-1976), dem ersten Präsidenten der St.-LukasGilde: Dieser wurde aus politischen Gründen im Mai 1938 als Direktor des Wiener St. Anna-Kinderspitals entlassen. Im Jahr 1940 wurde zudem seine Habilitation als ungültig erklärt. Während des Zweiten Weltkriegs arbeitete Orel als niedergelassener Pädiater in Wien [32, S. 396-397].

Letztlich wurden mehr als $10 \%$ Prozent der Gildenmitglieder von den Nazis verfolgt [26]. Diese Aspekte fanden bei Czech und Sheffer keine Erwähnung. Besonders bei Ersterem verwundert dies, dankte ihm doch die Historikerin Lö-

\footnotetext{
2 Universitätsarchiv Wien (UAW), Akademischer Senat, S. 127.9, Schreiben des Reichsführers SS und Chef der deutschen Polizei im Reichministerium des Innern an den Reichsminister für Wissenschaft, Erziehung und Volksbildung vom 05.11.1942.
}

scher in der bisher einzigen vorliegenden Gesamtdarstellung über die St.-Lukas-Gilde für seine Unterstützung als Berater bei der Erstellung ihrer Dissertation [25, S. 1].

\section{Hans Asperger und die Verfolgung durch die Gestapo}

Sagte Asperger die Unwahrheit, wie deduziert wird [11, S. 9, Anm. 40], als er in seiner Antrittsvorlesung in Wien erklärte, dass ihn der bekennende Nationalsozialist und Leiter der Wiener Universitätskinderklinik Franz Hamburger (1874-1954) „Zweimal mit persönlichem Einsatz und nicht geringer eigener Gefahr vor der Verhaftung durch die $\mathrm{Ge}$ stapo" gerettet hatte [2]? Asperger hat diese Aussage 1974 in einem Radiointerview wiederholt und die Angelegenheit bereits 1957 in seiner Antrittsvorlesung in Innsbruck anklingen lassen. Er sagte damals, er würde Hamburger nicht vergessen, dass dieser ihm „mit einem Einsatz, der ihn selbst gefährdete, die Freiheit und vielleicht das Leben rettete“ [5].

Zunächst stellten sich die Autoren die Frage, warum Asperger in dieser Angelegenheit hätte lügen sollen? Was wäre sein Vorteil gewesen, wenn er diese Aussage 


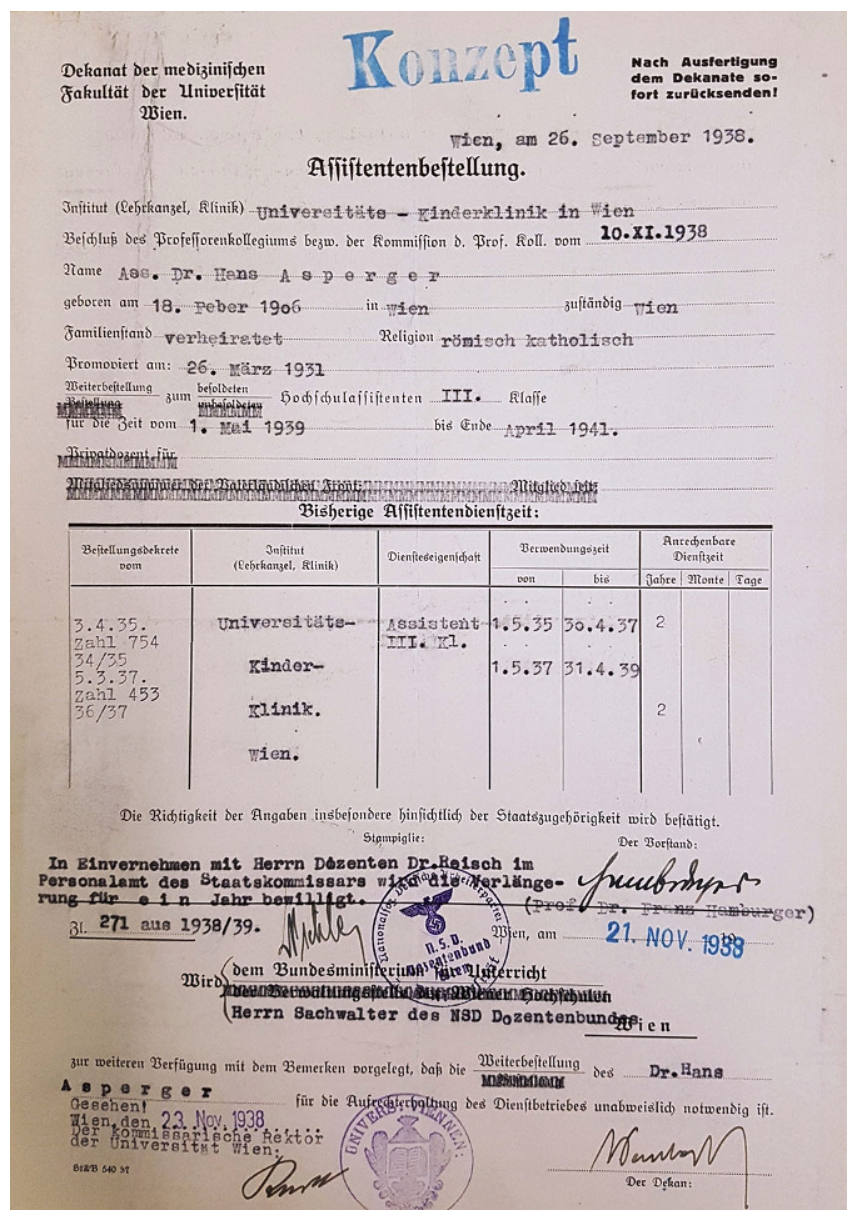

Reichsstatthalterei in Kenntnis gesetzt, dass gegen Asperger „keine Maßnahme zu treffen ist" ${ }^{\text {"5 }}$. Der Kurator der wissenschaftlichen Hochschulen in Wien genehmigte allerdings erst am 24.11.1940 die Verlängerung von Asperger rückwirkend als wissenschaftlichen Assistenten bis zum 30.04.1941. Welche Tätigkeit, Stellung oder Bezahlung Asperger vom Auslaufen seines Vertrags am 30.04.1939 bis zur rückwirkenden Anstellung am 24.11.1940 an der Kinderklinik hatte, ist aus der Personalakte nicht ersichtlich. ${ }^{6}$

》V Vermutlich nahm die Gestapo
Asperger wegen missachteter
Meldepflicht von erbkranken
Kindern ins Visier

Abb. $2<$ Dokumentation über die Weiterbestellung von Hans Asperger als Assistent an der Universitätskinderklinik Wien vom 26.09.1938. (UAW, Med. Fak., 271 aus 38/39)

vor den Spitzen der medizinischen Fakultät, der Universität Wien, den VertreterInnen von Politik und Kirche 1962 ohne Wahrheitsbezug getätigt hätte? Hamburger war zu den genannten Zeitpunkten bereits tot.

Die Autoren glaubten zunächst, die Aussage könnte seiner Befürchtung entsprungen sein, aufgrund seiner Mitgliedschaften beim Bund Neuland oder der St.-Lukas-Gilde in der Zeit vor 1938 ins Kreuzfeuer der Gestapo geraten zu sein. Tatsächlich wurde die Weiterbestellung von Hans Asperger als Assistent an der Kinderklinik nach dem 30.04.1939 auf 2 Jahre, obwohl von Hamburger am 26.09.1938 beantragt und vom Fakultätskollegium bereits beschlossen, durch den NSD-Dozentenbund und die Reichsstatthalterei beeinsprucht (ब Abb. 2). Bereits einen Monat nach dem Ansuchen um Weiterbestellung eröffnete die Reichsstatthalterei Wien [37], am Ballhausplatz residierend, ein Ermittlungsverfahren gegen Asperger entsprechend der „Verordnung zur Neuordnung des österreichischen Berufsbeamtentums" vom 31.05.1938. ${ }^{3}$ Hamburger einigte sich am 21.11.1938 mit dem Vertreter der Reichsstatthalterei vorerst auf eine Verlängerung um ein Jahr (• Abb. 2).

In den Folgemonaten zog die Reichsstatthalterei diverse Auskünfte über Asperger ein, wobei es auch um seine Tätigkeiten bei Neuland und der St.Lukas-Gilde und einiges mehr ging. ${ }^{4} \mathrm{Am}$ 07.06.1939 wurde der Rektor von der

\footnotetext{
3 "Die Verordnung ist das zentrale Instrument zur völkischen Neuordnung des öffentlichen Dienstes. $§ 3$ betrifft die Entfernung von Juden, jüdischen Mischlingen und jüdisch versippten Beamten aus dem Öffentlichen Dienst. § 4 verfügt die Pensionierung oderEntlassung von politisch unzuverlässigen Beamten bis 31.12.1938." http://ns-quellen.at/gesetz_anzeigen_detail. php?gesetz_id=28110\&action=B_Read.Zugegriffen:26. März 2020.

4 Österreichisches Staatsarchiv (ÖStA), Archiv der Republik (AdR), Gauakt 36055 Hans Asperger.
}

Die Aussagen von Asperger selbst [4] und die seiner Kinder [1] legen nach Meinung der Autoren nahe, dass Asperger wegen Unterlaufung der Meldepflicht von Kindern mit Erkrankungen, die unter das „Gesetz zur Verhütung erbkranken Nachwuchses“ vom 25. 07.1933 fielen, ins Visier der Gestapo gekommen sein dürfte. Diese Annahme wird nochmals gestützt durch die Stellungnahme von Christoph Groh, die bei den Zeitzeugenberichten in diesem Sonderheft zu finden ist. Tatsächlich wäre Asperger, der damaligen Gesetzeslage entsprechend, als Universitätsassistent verpflichtet gewesen, entsprechende PatientInnen zu melden. Nach einer diesbezüglichen Weisung vom 18.07.1940 wurde das Wiener Allgemeine Krankenhaus (AKH) im Juli 1942 von einem Vertreter des Hauptgesundheitsamtes, Abteilung Erb- und Rassenpflege, erneut auf diese Meldepflicht hingewiesen, nachdem bei einem Kind mit „doppelseitiger Hüftgelenkverrenkung" die Meldung von der Orthopä-

\footnotetext{
5 UAW, Med. Fak., 2201 aus 38/39, Schreiben der Reichsstatthalterei an den Rektor der Universität Wien vom 07.06.1939.

${ }^{6}$ ÖStA, AdR, Unterricht, PA 10/02 Hans Asperger, Schreiben des Kurators der wissenschaftlichen Hochschulen in Wien vom 24.11.1940.

7 WienerStadt-und Landesarchiv, 1.3.2.209.1.A3 - Direktionsakten: Schachtel 64 Mappe 114, Weisung vom 17.07.1940.
} 


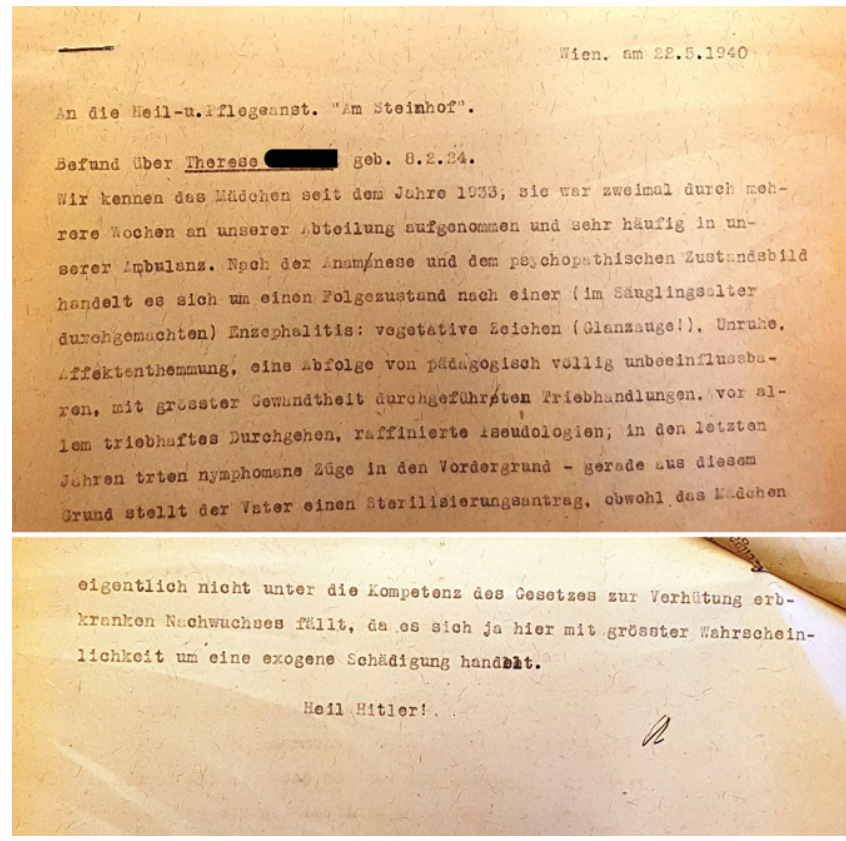

Abb. $3<$ Gutachten und Stellungnahme von Asperger vom 22.05 .1940 zur beabsichtigten Sterilisierung der Patientin Gertrude B., in der Stellungnahme fälschlicherweise "Therese" bezeichnet. (WStLA, 1.3.2.209.1.A47 Kinderklinik; Heilpädagogische Station: Krankengeschichten 1937, Gertrude B.)

die unterblieben war. ${ }^{7}$ In diesem Schreiben wird aber auch „das bewährte Meldeverfahren der psychiatrischen Universitätsklinik" lobend hervorgehoben. ${ }^{8}$

Wenn nun von der heilpädagogischen Station keine Meldungen, etwa über „Schwachsinnige“, kamen, könnte das natürlich Verdacht erregt haben. Asperger selbst gab jedenfalls an, dass er keine entsprechenden Meldungen gemacht habe [4], und bislang konnten solche von Asperger ausgefüllten auch nicht gefunden werden. In Springs ausführlicher Studie zur Zwangssterilisation in Wien zwischen 1940 und 1945 kommt der Name "Asperger" jedenfalls nicht vor [35].

\section{》) Asperger hat Gertrude \\ B. vor dem Zugriff der \\ Zwangssterilisation bewahrt}

Es gibt jedoch einige PatientInnen, die Asperger vor dem Zugriff der Zwangssterilisation bewahrt hat bzw. zu bewahren versuchte, wie etwa Gertrude B. (• Abb. 3, hier fälschlich als Therese bezeichnet).

Letztlich führte dieses Verhalten offenbar dazu, dass es sowohl seinem

\footnotetext{
8 WienerStadt-undLandesarchiv, 1.3.2.209.1.A3 - Direktionsakten: Schachtel 64 Mappe 114, Schreiben vom 17.07.1942.
}

Protektor Hamburger als auch Asperger selbst zu gefährlich erschien, in dieser Art fortzufahren. Die Lösung: Asperger musste 1943 in den Krieg ziehen. Er sagte dazu 1974 in seinem Radiointerview: „Schließlich war es aber für beide Teile [gemeint sind Asperger und Hamburger; Anm. d. Autoren] das Beste, wenn Doktor Asperger, schließlich dann schon Dozent für Kinderheilkunde, einrückte“ [4]. Tatsächlich wurde der Antrag auf Verlängerung seiner Unabkömmlichstellung (UK-Stellung) am 09.03.1943 abgelehnt. ${ }^{9}$ Auf Hamburgers Protest hin wurde dieser informiert, dass die Einberufung Aspergers durch die ,verantwortlichen staatlichen Funktionäre" erfolgt sei und nicht durch die Wehrersatzinspektion. ${ }^{10}$

\footnotetext{
9 UAW, Akademischer Senat, S 133/1 (U.K. Hilfsakte, 1939-45), Schreiben des Rektors Knoll an die Wehrbezirkskommandanten vom 24.01.1940. UAW, Akademischer Senat, S 133/3 (U.K. Akten 1-100, 1939-45), Gesch.-Z: 24, UKKarte von Asperger Dr., Johann. Ab Januar 1940 war die Universität Wien berechtigt, Personen namhaft zu machen, die für den Betrieb der Universität unabkömmlich waren (UK-Stellung). Diese Personen waren in der Folge auf Zeit von der Einberufung zur Deutschen Wehrmacht befreit. Eine Verlängerung war möglich.

10 UAW, Akademischer Senat, S 133/3 (U.K. Akten 1-100, 1939-45), Gesch.-Z: 24, Schreiben der Wehrersatzinspektion vom 19.03.1943.
}

Am 25.03.1943 musste Asperger nach Brünn einrücken. ${ }^{11}$

Diese Archivalien wurden in bisherigen Publikationen nach Ansicht der Autoren nur unvollständig wiedergegeben [11, S. 8-11]. Wäre eine komplette Auflistung und gesamtheitliche Interpretation nicht notwendig, um die Hintergründe von Aspergers angeblicher Kollaboration mit dem NS-Regime genauer zu beleuchten? Detailliertere Darstellungen u. a. zu den vertraglichen Schwierigkeiten Aspergers, der St.-Lukas-Gilde und $\mathrm{zu}$ Aspergers UK-Stellung verdienen jedenfalls Berücksichtigung und befinden sich deswegen gerade in Vorbereitung.

\section{Reaktion der SchülerInnen und KollegInnen}

Zwischen den Darstellungen in den erwähnten Publikationen [11,33] und den in diesem Beitrag präsentierten Archivalien sind, wie oben ausgeführt, unvereinbare Unterschiede zu verzeichnen. Dies und auch eigene Erfahrungen mit der Person Hans Aspergers veranlassten einige seiner SchülerInnen und KollegInnen, ab dem Sommer 2018 in unregelmäßigen Abständen Treffen mit Historikern abzuhalten, relevante Dokumente aus Archiven zu besorgen und gemeinsam mit Historikern ein eigenständiges Bild von dieser Zeit und Asperger zu erarbeiten. Erste Ergebnisse finden sich z. T. bereits in diesem Heft; weitere Narrative sind in Vorbereitung. Besonderes Augenmerk wird auf die Umstände bei der Überweisung der Kinder Herta und Elisabeth Schreiber auf den Spiegelgrund und die Begutachtung der Kinder durch die Gugging-Kommission gelegt.

Bereits im Herbst 2018 einigten sich 21 der ehemaligen SchülerInnen und AssistentInnen von Asperger darauf, einen Leserbrief an Molecular Autism zu senden, der eigene Erfahrungen mit der Person Aspergers darstellen und einige der oben gezeigten Unterschiede aufzeigen sollte (• Abb. 4).

Am 16.01.2019 erfolgte die Ablehnung des Leserbriefs: „After discussing the EB

11 ÖStA, AdR, Unterricht, PA 10/02 Hans Asperger, Personalakt Asperger, Meldung von Franz Hamburgervom 25.03.1943. 
Dear Editor,

After reading Herwig Czech's article and the editorial on Hans Asperger published in this journal on April 19, 2018, we previous students and colleagues of Asperger - were first shocked and speechless. Is it possible that Asperger was in "close alliance with, and support of, the Nazi program of compulsory sterilization and euthanasia" and we did not realize? Could he hide so artfully his true nature as a willing "cog in the Nazi killing machine" for decades?

Why did we not realize what Herwig Czech claims to prove? Should the picture painted by H. Czech and the editors of this journal be real, then several severe breaks in Asperger's personality must have taken place.

Our personal experience with Hans Asperger during our medical education dates from the sixties and seventies of the last century, when he was chair of the Department of Paediatrics in Innsbruck and later in Vienna. We attended his lectures, did our internship, residency or made our first scientific steps in the clinic headed by him. Although some of us found his general paediatric knowledge rather limited, we were impressed by his liberal, humanitarian and respectful approach to children, his patients and their parents. We never observed any dismissive remarks or - even worse - evidence for national-socialistic, eugenic ideologies at presentations or discussions of patients, still with severe disabilities. In general, his attitude towards others (including his rivals) was rather mild, paternal and nuanced. He hardly tried to press or force someone of us to something we did not want to. Therefore, he was often criticized by others as being "too weak" for a department chair.

For our generation of paediatricians, his "Literary Readings" at the annual Paediatric Education Programme in the ski resort Obergurgl are unforgettable. They showed the true spirit of a "Heilpädagoge" convinced that confrontation with good literature would educate participants to more humanitarian beings.

Clearly, our personal experience with Hans Asperger does not allow final conclusions on his behaviour during the NS-time. But: Does Czech's article contain enough evidence to ultimately sentence him?

No. Not only are some of Czech's statements pure speculations. There is also the fact that other - heavy weight! - data not fitting into the picture are simply not presented in the publication.

Let us just mention two facts proving this:

In March 1938 Dr. Carla Zawisch, secretary and driving force of the catholic St.-Lukas-Gilde was imprisoned, deprived of her "Habilitation" and position at the University of Vienna and finally forced to emigrate to the USA by the Nazis. The reason for this was her activity at the board of the Gilde and the Gilde's catholic stance to euthanasia contrary to the Nazi-Ideology [11].

A similar fate had the first president of the Gilde, Herbert Orel [12]

Thus, being also a member of the board of the Gilde, why should Asperger "in 1938 found enough common ground with National Socialism to establish himself as a credible fellow traveller in the eyes of the party, without directly embracing National Socialism" [1] as Czech claims.

Why was Erwin Hesse, cochair with Asperger at the "Fahrenden Scholaren", a group of the Bund Neuland, imprisoned 1939 by the Gestapo and banned from Vienna [10], if this group was "part of the Bund's decidedly völkisch and right-wing faction", as Czech insists upon?

The fate of these 3 colleagues, with whom he shared the engagement in "Gilde" and "Scholaren", was reason enough to be afraid to be the next to be "interrogated" by the Gestapo (even more so, as Asperger at the time was married and had two small children, so he had to be afraid for his young family, too).

But Czech interprets Asperger's fears as insubstantial lies to cover himself. What is his evidence?

If we follow Asperger's narrative, in 1943 he decided together with Franz Hamburger, his mentor and teacher - a "convinced NAZI" according to Asperger -,- to enlist, because the pressure at the Kinderklinik became unbearably dangerous [ Footnote 10].

There are many open questions, i.e. why was Asperger so generously supported by Franz Hamburger? Why precisely were the children Herta and Elisabeth Schreiber transferred to Spiegelgrund? What should be the rational sending these children to euthanasia without notifying Berlin as NS-law required? etc.

We started to recheck data presented by Herwig Czech and will go into details. It may take some time, most of us are 70 years and more, but we hope we will then get the chance to present a different picture with more data and less interpretations and speculations.

Deutsch Johann MD, assoc. Prof., Paed. Gastroenterology

Frisch Herwig MD, assoc. Prof., Paed. Endocrinology

Graf Grete MD, General Medicine

Graf Martin MD, assoc. Prof., Neurology

Granditsch Gerhard MD, assoc. Prof., Paed. Gastroenterology

Groh Christoph MD, assoc. Prof., Paed. Epileptology

Hammerer Ignaz MD, assoc. Prof., Paed. Cardiology

Jaschke Erna MD, Dermatology

Jürgenssen Olaf Arne MD, General Paediatrics

Katschnig Hildegard MD, ass. Prof., General Paediatrics \& Family Therapy

Kiss Irmgard MD, General Paediatrics

Krisper Heinz MD, Neuropaediatrics

Kurz Ronald MD, em. Prof. of Paediatrics

Kurz Ronald MD, em. Prof. of Paediatrics

Popow Christian MD, assoc. Prof., Child
Rath Franz MD, General Paediatrics

Rath Franz MD, General Paediatrics
Rosenmayr Franz MD, assoc. Prof., Paed. Neurology tt

Stiskal Alfred MD, General Paediatrics

Scheminzky Christine MD, General Paediatrics

Tatzer Ernst MD, Child Psychiatry

Waldhauser Franz, MD, assoc. Prof., Paed. Endocrinology

Widhalm Kurt MD, em. Prof. of Paediatrics, Nutrition-Lipid Disorders-Obesity

\# deceased in April 2019

Abb. $4<$ Leserbrief der 21 ehemaligen SchülerInnen und AssistentInnen von Asperger an Molecular Autism 
[Editorial Board; Anm.d. Autoren] members decided to withdraw your manuscript and suggest to submit it to a journal that specializes more in history"12. Die Begründung erstaunte insofern, als das Journal ein halbes Jahr vorher die eindeutig historisch ausgerichtete 43 Seiten lange Arbeit von Czech publiziert hatte, der die Tätigkeit von Hans Asperger in der Zwischenkriegszeit und nach dem sog. Anschluss zum Gegenstand hat und diese in den historischen Kontext der zentraleuropäischen Geschichte stellt. Zudem wurde im Editorial von Molecular Autism aus dem April 2018 angekündigt, dass es das Ziel des Journals sei „to publish excellent research into autism of any kind, whether the research focuses on the molecular, neurological, psychological, clinical, or in this case social aspects" [7].

\section{》) Der Gegendarstellung in Molecular Autism wurde von dessen Herausgeberlnnen kein Platz eingeräumt}

Die Verfasser des Leserbriefs reagierten mit folgender Nachricht: „We have all sympathy for an EB specialized in molecular biology who does not feel competent to publish on Central European history. However, you published a 43-page report on Hans Asperger in the Nazi-time which effectively ruined his reputation. But you are not willing to allocate adequate space for presenting facts that show that this report's arguments are manipulative. ${ }^{13}$ This is neither fair nor does it serve scientific truth $^{\text {"14. }}$.

Diese Nachricht blieb bis heute unbeantwortet. Nur der US-amerikanische Autor Silberman, der Verfasser des Bestsellers über Autismus Geniale Störung [34], einer der Mitverfasser des erwähn-

\footnotetext{
12 E-Mail von Samuel L. Membrano Jr. an die VerfasserInnen des Leserbriefes vom 16.01.2019.

13 Der Verfasser der E-Mail entschuldigt sich für die Verwendung des Ausdrucks "manipulative“ und überlässt die Bewertung der zitierten Arbeit dem/der Leserln.

${ }^{14}$ E-Mailvon den Verfasserlnnen des Leserbriefs an Samuel L. Membrano Jr. vom 29.01.2019.
}

ten Editorials, aber nicht Mitglied des Editorial Board von Molecular Autism, dem die Nachricht in Kopie zugeschickt wurde, reagierte innerhalb weniger Stunden: Er sei mit der zurzeit laufenden Aktion gegen Asperger nicht einverstanden, er halte es mit dem bisher vorliegenden Material für verfrüht, den Stab über Asperger zu brechen. Silberman vermittelte den Kontakt zur US-amerikanischen Anthropologin Falk, deren Publikation über Asperger mit praktisch wortidentischer Begründung wie der oben genannte Leserbrief von Molecular Autism zurückgewiesen wurde. Ihre Publikation ist inzwischen unter dem Titel: „Non-complicit: revisiting Hans Asperger's career in Nazi-era Vienna“ im Journal of Autism and Developmental Disorders [15] erschienen und hat zu Reaktion [12] und Gegenreaktion [14] geführt.

\section{Schlussbemerkung}

Viele der noch lebenden SchülerInnen, AssistentInnen und KollegInnen Aspergers sind interessiert, ein möglichst wahrheitsgetreues Bild über ihn zu bekommen. Die Autoren des vorliegenden Beitrags werden ihren Teil dazu beitragen, sicher auch geprägt durch die eigenen Begegnungen mit ihm, aber auch seiner Aussage über einen von ihm geschätzten Wissenschaftler folgend ${ }^{15}$ : „Ihn hat immer interessiert, wie der liebe Gott die Welt gemacht hat". Für mehrere der SchülerInnen und KollegInnen ist dieser Satz ein lebenslanges Vermächtnis geblieben.

\section{Korrespondenzadresse}

Univ.-Prof. Dr. med. univ. Franz Waldhauser Amerlingstr. 4/20, 1060 Wien, Österreich f.waldhauser@aon.at

Univ.-Prof. Dr. med. univ. Franz Waldhauser, geb. 1946. Eintritt Univ.-Kinderklinik Wien 1976. Facharzt für Kinderheilkunde 1981. Advanced Postdoc Fellow MIT (Boston) 1981-1983. Visiting Scientist an der Harvard Medical School (Boston) 1990-1991. Stellv. Leiter der Allg. Pädiatrie, Allgemeines Krankenhaus (AKH), Wien 1997-2003. Herausgeber des Newsletters Schwerpunkte und Netzwerke für Seltene Erkrankungen

15 Otto Thalhammer (1922-1994), Prof. für Kinderheilkunde, Neonatologe, 1966 Implementation des österreichweiten Neugeborenenscreenings auf angeborene Stoffwechselerkrankungen. seit 2017. Medizinisch-wissenschaftliche Tätigkeit: päd. Endokrinologie, Störungen der Geschlechtsentwicklung, Melatonin. Med.-polit. Tätigkeit: stellv. Kuriensprecher des Mittelbaues, stellv. Leiter des Strukturteam-AKH, Mitbegründer der Politischen Kindermedizin.

Univ.-Prof. Dr. med. univ. Johann Deutsch, geb. 1944. Ausbildung an der Univ.-Kinderklinik Wien 1972-1978. Studienaufenthalte in Zürich, Melbourne, London, Pittsburgh und Hannover 1980-1985. Leiter der Arbeitsgruppe für pädiatrische Hepatologie an der Univ.-Kinderklinik Graz 1979-2009. Gründung und Vorstandsmitglied des Forschungsvereins inVita 1988-2019. Vorstandsmitglied und Vorsitzender der GPGE 1991-1995. Mitglied der Ernährungskommission der ÖGKJ 1994-2009. Leitung des GPGEzertifizierten Ausbildungszentrums für Pädiatrische Gastroenterologie, Hepatologie und Ernährung an den Univ.-Kliniken für Kinder- und Jugendheilkunde und Kinder- und Jugendchirurgie Graz 2003-2009.

Univ.-Prof. Dr. med. univ. Christian Popow, MSc, geb. 1950. Studium der Sozial- und Wirtschaftswissenschaften (1. Studienabschnitt), und Medizin (Facharzt für Kinder- und Jugendheilkunde und -psychiatrie) und Psychotherapeut (kognitive Verhaltenstherapie). Tätigkeit an der Univ.-Klinik für Kinder- und Jugendheilkunde bzw. Kinder- und Jugendpsychiatrie, derzeit am Landesklinikum Mauer. Lehr- und Supervisionstätigkeit. Wissenschaftliche Schwerpunkte: Neonatologie und Intensivmedizin, angewandte Computerwissenschaften, Kinder- und Jugendpsychiatrie und Psychotherapie.

\section{Einhaltung ethischer Richtlinien}

Interessenkonflikt. F. Waldhauser, J. Deutsch und C. Popow geben an, dass kein Interessenkonflikt besteht.

Für diesen Beitrag wurden von den Autoren keine Studien an Menschen oder Tieren durchgeführt. Für die aufgeführten Studien gelten die jeweils dort angegebenen ethischen Richtlinien.

\section{Literatur}

1. Asperger Felder M (2020) Notariell beglaubigte Mitteilung von Dr. Maria Asperger Felder

2. Asperger H (1978) Antrittsvorlesung des österreichischen Kinderarztes und Heilpädagogen Univ.Prof. Hans Asperger - "Ecce in focus" - Wien 10.10.1962 ( $\min 7: 30$ bis 8:15). https://www. mediathek.at/portaltreffer/atom/12FE48A0-3B200160-00000AD8-12FD8E35/pool/BWEB/. Zugegriffen: 24. März 2020

3. Asperger H (1978) Geschichten und Geschichte - Autobiographische Aussagen von Hans Asperger. Radiointerview mit Hans Asperger vom 24.12.1974 (min 22:30-27:00). https://www.mediathek.at/atom/01782B100D9-00CD5-00000BEC-01772EE2. Zugegriffen: 24. März 2020

4. Asperger H (1978) Geschichten und Geschichte - Autobiographische Aussagen von Hans Asperger. Radiointerview mit Hans Asperger vom 24.12.1974 ( $\min 38: 00$ bis 42:00). https://www.mediathek.at/atom/01782B100D9-00CD5-00000BEC-01772EE2. Zugegriffen: 24. März 2020 
5. Asperger H (1957) Probleme der modernen Pädiatrie. Wien Klin Wochenschr 69:549-553

6. Asperger H (1934) Spruch. Neue Jugend 16:20-23

7. Baron-Cohen S, Klin A, Silberman S et al (2018) Did Hans Asperger actively assist the Nazi euthanasia program? Mol Autism 9:28

8. Behal B (2009) „Kontinuitäten und Diskontinuitäten deutschnationaler katholischer Eliten im Zeitraum 1930-1965" Ihr Weg und Wandel in diesen Jahren am Beispiel Dr. Anton Böhms, Dr. Theodor Veiters und ihrer katholischen und politischen Netzwerke. Dissertation. Universität Wien, Wien

9. Cooke P (2018) Hans Asperger 'helped Nazis murder dozens of disabled kids during World War Two' before he identified autism. https://www.thesun.co.uk/news/6089118/hansasperger-autism-doctor-helped-nazis-murderchildren/.Zugegriffen: 24. März 2020

10. Czech H (2014) Der Spiegelgrund-Komplex. Kinderheilkunde, Heilpädagogik, Psychiatrie und Jugendfürsorge im Nationalsozialismus. Österr Z Geschichtswiss 25:194-219

11. Czech H (2018) Hans Asperger, National Socialism, and "race hygiene" in Nazi-era Vienna. Mol Autism 9:1-43

12. Czech H (2019) Response to 'non-complicit: revisiting Hans Asperger's career in Nazi-era Vienna.J Autism Dev Disord 49:3883-3887

13. Donvan J (2018) Why did it take so long to expose Hans Asperger's Nazi ties? https://www. theatlantic.com/science/archive/2018/04/whyit-took-so-long-to-expose-hans-aspergers-nazities/558872/.Zugegriffen: 24. März2020

14. Falk D (2019) More on Asperger's career: a reply to Czech. J Autism Dev Disord 49:3877-3882

15. Falk D (2019) Non-complicit: revisiting Hans Asperger's career in Nazi-era Vienna. J Autism Dev Disord. https://doi.org/10.1007/s10803-01903981-7

16. Gander K (2018) Autism Doctor Hans Asperger 'linked to Nazi murder of disabled children. https://www.newsweek.com/autism-hansasperger-nazi-murder-disabled-children-newstudy-892368. Zugegriffen:24. März 2020

17. Gemelli A (1936) Grußbotschaft von Papst Pius XI. St. Lukas. Mitt Österr St Lukas Gilde 4:73-76

18. Hager C (2014) Hans Asperger - „Er war Teil des Apparats". https://www.wienerzeitung. at/dossiers/autismus/618698_Erwar-Teil-desApparats.html?em_no_split=1. Zugegriffen: 24 . März 2020

19. Heer F (2001) Der Kampf um die österreichische Identität. Böhlau, Wien

20. Hesse E Gelebtes und Gedachtes. Unpublizierte Aufsatzsammlung. In: Diözesanarchiv Wien, Nachlass Erwin Hesse.

21. Kapfhammer FM (1987) Neuland. Erlebnis einer Jugendbewegung. Styria, Graz Wien Köln

22. Kernbauer A (1996) Carla Zawisch-Ossenitz. Eine biografische Skizze der ersten Professorin der Grazer Universität. In: Kernbauer A, LienhartSchmidlechner K (Hrsg) Frauenstudium und Frauenkarrieren an der Universität Graz. Akademische Druck-u. Verlagsanstalt, Graz, S265-270

23. Kleinert D (2010) Willibald Pahr: Außenminister unter Kreisky. Analysen zum Zeitgeschehen. Böhlau, Wien

24. Kuchar L (2020) Ein „Spinner" auf Spurensuche. http://www.schritte-des-glaubens.at/spinner. pdf.Zugegriffen: 24. März 2020

25. Löscher M (2005) „.... der gesunden Vernunft nicht zuwider ..."? Eugenik in katholischen Milieus/ Netzwerken in Österreich vor 1938. Dissertation. Universität Wien, Wien
26. Löscher $M(2009)$ „.... der gesunden Vernunft nicht zuwider ..."? Katholische Eugenik in Österreich vor 1938. StudienVerlag, Innsbruck Wien Bozen

27. Pernkopf E (1943) Schreiben des Rektors vom 11.09.1943. Universitätsarchiv Wien, Akademischer Senat, Wien, S127.9.

28. Preidt R (2018) Doctor who gave name to Asperger's syndrome worked with Nazis. https:// health.usnews.com/health-care/articles/201804-19/doctor-who-gave-name-to-aspergerssyndrome-worked-with-nazis-report. Zugegriffen:24. März 2020

29. Rennert D (2018) Autismusforscher Hans Asperger schickte Kinder in den Tod. https:// www.derstandard.at/story/2000078268222/ autismusforscher-hans-asperger-schicktekinder-in-den-tod.Zugegriffen:24. März 2020

30. Seewann G (1971) Die Entstehung der Deutschen Jugendbewegung in Österreich-Ungarn 1900 bis 1914 und die Fortsetzung in ihrem katholischen Zweig "Bund Neuland" von 1918 bis 1938 Österreichische Jugendbewegung 1900 bis 1938. Bd. 1.Dipa-Verlag, Frankfurtam Main

31. Seewann G (1971) Die Entstehung der Deutschen Jugendbewegung in Österreich-Ungarn 1900 bis 1914 und die Fortsetzung in ihrem katholischen Zweig „Bund Neuland" von 1918 bis 1938. Österreichische Jugendbewegung 1900 bis 1938 , Bd. 2. Dipa-Verlag, Frankfurt am Main

32. Seidler E (2007) Jüdische Kinderärzte 1933-1945. Entrechtet/Geflohen/Ermordet. Karger, Basel Freiburg

33. Sheffer E (2018) Asperger's children: the origins of autism in Nazi Vienna. Norton, New York

34. Silberman S (2017) Geniale Störung. Die geheime Geschichte des Autismus und warum wir Menschen brauchen, die anders denken. DuMont, Köln

35. Spring CA (2009) Zwischen Krieg und Euthanasie - Zwangssterilisation in Wien 1940-1945. Böhlau, Wien Köln Weimar

36. Steininger R (2005) Der Staatsvertrag. Österreich im Schatten von deutscher Frage und Kaltem Krieg 1938-1955. Studienverlag, Innsbruck

37. Wächter O (1938) Schreiben des Staatskommissars beim Reichsstatthalter SS-Standartenführer Dr. Otto Wächter an Gauleitung Wien vom 24.10.1938. In: Österreichisches Staatsarchiv, Archiv der Republik, Gauakt 36055 Hans Asperger

38. Walters G (2018) How DID Dr Asperger hide his past as a CHILD-KILLING Nazi monster for so long? https://www.dailymail.co.uk/news/article5636431/GUY-WALTERS-DID-Dr-Asperger-hidepast-CHILD-KILLING-Nazi-monster-long.html. Zugegriffen: 24. März 2020

39. Wodrazka PB (2020) Prälat Hesse, ein bis heute unvergessener Seelsorger - kurze Lebensbeschreibung des langjährigen Pfarrers von St. Rochus. http://www.rochuskirche.at/ oratorium/geschichte/das-oratorium-in-wien/ praelat-hesse-ein-bis-heute-unvergessenerseelsorger/.Zugegriffen: 24. März 2020

40. Zawisch C (1955) Brief von Carla Zawisch. Diözesanarchiv Wien, Wien (Nachlass Karl Ludwig I/2.)

41. Zawisch C (1933) Was wir sein, was wir tun wollen. St. Lukas. Mitt Österr St Lukas Gilde 1:2-3

42. Molecular Autism (Hrsg) (2018) Herwig Czech "Hans Asperger, National Socialism, and 'race hygiene' in Nazi-era Vienna". https:// molecularautism.biomedcentral.com/articles/10 1186/s13229-018-0208-6. Zugegriffen: 19. Apr. 2018

43. Molecular Autism (Hrsg) (2018) Editorial: Did Hans Asperger actively assist the Nazi euthanasia pro- gram? https://molecularautism.biomedcentral. com/articles/10.1186/s13229-018-0209-5. Zugegriffen: 19. Apr. 2018

44. The Guardian (Hrsg) (2018) Hans Asperger aided and supported Nazi programme, study says. https://www.theguardian.com/world/2018/apr/ 19/hans-asperger-aided-and-supported-naziprogramme-study-says?CMP=fb_gu. Zugegriffen: 18. Apr. 2018

45. New York Times (Hrsg) (2018) Hans Asperger aided Nazi child euthanasia, study says. https://www. nytimes.com/2018/04/19/world/europe/hansasperger-nazis.html.Zugegriffen: 19. Apr. 2018

46. Washington Post (Hrsg) (2018) Hans Asperger, hailed for autism research, may have sent child patients to be killed by Nazis. https:// www.washingtonpost.com/news/retropolis/wp/ 2018/04/19/hans-asperger-hailed-for-autismresearch-may-have-sent-child-patients-to-bekilled-by-nazis/?utm_term=.d59b565afbd0. Zugegriffen: 19. Apr. 2018

47. Telegraph (Hrsg) (2018) Pioneer doctor Asperger was Nazi collaborator who 'sent children to their deaths. https://www.telegraph.co. uk/news/2018/04/19/aspergers-pioneer-nazicollaborator-sent-children-deaths/. Zugegriffen: 19. Apr. 2018

48. Stuff (New Zealand) (Hrsg) (2018) Child doctor Hans Asperger 'willingly became cog in the Nazi killing machine. https://www.stuff. co.nz/world/europe/103257407/child-doctorhans-asperger-willingly-became-cog-in-thenazi-killing-machine.Zugegriffen: 19. Apr. 2018

49. SBS News (Australia) (Hrsg) (2018) Austrian paediatrician Hans Asperger reportedly ",actively cooperated" with the Nazi euthanasia programme. https://www.sbs.com.au/news/hans-aspergeractively-cooperated-with-nazis. Zugegriffen: 20. Apr. 2018

50. South China Morning-Post (Hrsg) (2018) Famed paediatrician Hans Asperger 'actively cooperated' with Nazis during second world war, according to new research. https://www. scmp.com/news/world/europe/article/2142458/ famed-paediatrician-hans-asperger-activelycooperated-nazis-during. Zugegriffen: 19. Apr. 2018

51. Folha de S.Paulo (Brasil) (Hrsg) (2018) Asperger colaborou com programa de eutanásia nazista, diz estudo. https://www1.folha.uol.com.br/ciencia/ 2018/04/asperger-colaborou-com-programade-eutanasia-nazista-diz-estudo.shtml.Zugegriffen: 19. Apr. 2018

52. Die Zeit (Hrsg) (2018) Hans Asperger: Ein guter Nazi? https://www.zeit.de/2018/17/ hans-asperger-kinderarzt-nationalsozialismusautismus-forschung-schuld/komplettansicht. Zugegriffen: 19. Apr. 2018

53. Neue Zürcher Zeitung (Hrsg) (2018) Hans Asperger soll Kinder an die Nazis ausgeliefert haben. https:// www.nzz.ch/wissenschaft/hans-asperger-sollmit-den-nazis-kooperiert-haben-ld.1378927. Zugegriffen: 19. Apr. 2018

54. Der Standard (Hrsg) (2018) Autismusforscher Hans Asperger schickte Kinder in den Tod. https://derstandard.at/2000078268222/ Autismusforscher-Hans-Asperger-schickteKinder-in-den-Tod.Zugegriffen: 19. Apr. 2018

55. Die Presse (Hrsg) (2018) Asperger half der NS-Euthanasie. https://diepresse.com/ home/science/5409660/Asperger-half-derNSEuthanasie.Zugegriffen:20. Apr. 2018

56. Sheffer E (2018) Asperger's children: the origins of autism in Nazi Vienna 